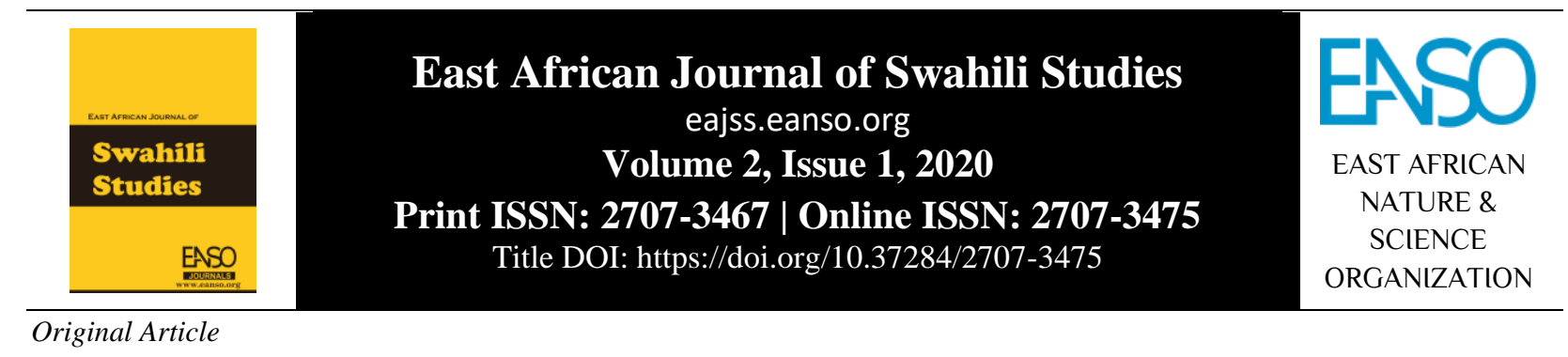

\title{
Umuhimu Wa Kufundisha Lugha Ya Kiswahili Katika Shule Za Sekondari Nchini Uganda
}

\author{
Dr. Willy Wanyenya ${ }^{1 *}$ \\ ${ }^{1}$ Makerere University Business School, P. O. Box 1337, Kampala, Uganda. \\ *Correspondence email: willywanyenya@gmail.com
}

Article DOI: https://doi.org/10.37284/eajss.2.1.138

Date Published: IKISIRI

14 Apr 2020 Katika makala haya, mtafiti anaeleza namna ambavyo Kiswahili kinaweza kuwa na manufaa kwa nchi ya Uganda. Ni kweli kwamba nchini Uganda, watu

Keywords: kadhaa wakiwemo baadhi ya wanasiasa hawaoni umuhimu wa lugha ya Kiswahili. Hata hivyo, nchi ya Uganda inaweza kunufaika pakubwa sana hasa Uchumi, kiuchumi kutokana na lugha ya Kiswahili. Ni jambo la wazi kuwa uchumi Lugha, duni hufanya nchi ikose kupata maendeleo ilhali uchumi imara hufanya nchi Maendeleo, Hafifu, Imara. iwe na maendeleo. Nchi zenye nguvu kama vile Marekani na zile za Ulaya zimeweza kupata maendeleo makubwa kwa sababu ya kuwa na uchumi imara. Kwa hiyo, nchi mbalimbali huweka mikakati maalumu ya kuimarisha uchumi zao. Nchi huwa zinaimarisha uchumi zaokwa kuimarisha sekta za biashara, kilimo, viwanda, uvuvi na utalii. Halikadhalika, nchi nyingi hutenga kiasi kikubwa cha pesa katika bajeti zao za kila mwaka wa kifedha kwa ajili ya kuimarisha sekta mbalimbali zinazoimarisha uchumi. Nchi mbalimbali pia huendeleza sekta ya kibinafsi katika harakati zao za kuimarisha uchumi na kuondoa umaskini katika jamii. Kwa hiyo, watu binafsi huwa wanahamasishwa kuanzisha miradi mbalimbali na kubuni kazi kwa wananchi badala ya kusubiri serikali. Hata hivyo, nchini Uganda, wanasiasa hawajaona umuhimu wa lugha ya Kiswahili na nchi hii ni mwanachama wa jumuiya ya Afrika Mashariki. Kwa hiyo, katika makala hii, mtafiti anaeleza jinsi Kiswahili kinaweza kuwa na manufaa kwa Waganda. Utafiti huu ulikusudia kujibu swali; Je, Kiswahili kinaweza kuwa na manufaa yoyote kwa nchi ya Uganda? Katika kazi hii, mkabala wa utafiti ulikuwa wa nyanjani. Mtafiti aliendeleza utafiti katika Wilaya ya Bududa nchini Uganda. Wahojiwa walikuwa walimu katika shule za sekondari. Taarifa zilikusanywa kutoka kwa walimu 100. Baada ya taarifa kukusanywa, mtafiti alizichanganua data hizo na kubainisha hoja tofautitofauti. Umuhimu wa utafiti huu ni kuwa ulipelekea 
wananchi wa Uganda kuona umuhimu wa lugha ya Kiswahili na hivyo kuanza kuitetea badala ya kuipiga vita.

\section{APA CITATION}

Wanyenya, W. (2020). Umuhimu Wa Kufundisha Lugha Ya Kiswahili Katika Shule Za Sekondari Nchini Uganda. East African Journal of Swahili Studies, 2(1), 19-27. https://doi.org/10.37284/eajss.2.1.138.

\section{CHICAGO CITATION}

Wanyenya, Willy. 2020. "Umuhimu Wa Kufundisha Lugha Ya Kiswahili Katika Shule Za Sekondari Nchini Uganda”. East African Journal of Swahili Studies 2 (1), 19-27. https://doi.org/10.37284/eajss.2.1.138.

\section{HARVARD CITATION}

Wanyenya, W. (2020) "Umuhimu Wa Kufundisha Lugha Ya Kiswahili Katika Shule Za Sekondari Nchini Uganda", East African Journal of Swahili Studies, 2(1), pp. 19-27. doi: 10.37284/eajss.2.1.138.

\section{IEEE CITATION}

W. Wanyenya, "Umuhimu Wa Kufundisha Lugha Ya Kiswahili Katika Shule Za Sekondari Nchini Uganda”, EAJSS, vol. 2, no. 1, pp. 19-27, Apr. 2020.

\section{MLA CITATION}

Wanyenya, Willy. "Umuhimu Wa Kufundisha Lugha Ya Kiswahili Katika Shule Za Sekondari Nchini Uganda". East African Journal of Swahili Studies, Vol. 2, no. 1, Apr. 2020, pp. 19-27, doi:10.37284/eajss.2.1.138.

\section{UTANGULIZI}

Lugha ni muhimu sana katika maisha ya binadamu. Lugha ni mojawapo ya zawadi kutoka kwa Mwenyezi Mungu. Hii ni kwa sababu binadamu hawezi akanunua wala akatengeneza lugha kama bidhaa. Lugha huwa inatekeleza mambo kadhaa wa kadhaa katika maisha ya binadamu. Lugha inaweza kuzua migogoro baina ya watu au baina ya nchi ikitumiwa vibaya au inaweza kuleta maafikiano miongoni mwa jamii au baina ya nchi ikitumiwa vizuri. Lugha hupelekea mambo mbalimbali kutekelezwa. Bila lugha, huenda binadamu asingekuwa na uwezo wa kuwasiliana kama anavyofanya sasa na kutekeleza yale anayoyatekeleza. Hivyo basi, binadamu ana uwezo wa kufanya jambo hili na lile kwa sababu ya kutumia lugha. Bila shaka, lugha ni nguzo muhimu katika nyanja za kisiasa, kiuchumi na kijamii. Hata hivyo, katika makala haya, mtafiti anaeleza mchango wa lugha ya Kiswahili katika ukuzaji wa uchumi wa nchi. Mtafiti alitimiza wajibu huu kwa kukutana na wahojiwa mbalimbali na kukusanya taarifa kuhusu hoja zinazolengwa na utafiti.

\section{SUALA LA UTAFITI}

Kwa mujibu wa kikao cha Bunge la Uganda mnamo Februari 2020, serikali ya Uganda iliamua kuanzisha rasmi mtalaa mpya wa elimu unaosisitiza somo la Kiswahili kuwa la lazima katika shule za sekondari kote nchini. Wabunge walipinga vikali sana na kuamrisha uzinduzi huo usitishwe mara moja. Wao hawakutaka Kiswahili kifanywe somo la lazima kwa wanafunzi, wakidai kuwa Waganda wengi huwa wanazungumza lugha ya Kiswahili bila hata kuhudhuria shule. Badala ya Kiswahili, wabunge hao walitaka lugha ya Kichina na Kiarabu zifanywe masomo ya lazima. Waliongeza kuwa Waganda wengi huwa wanafanya biashara Uchina na vijana wengi huwa wanaenda kwa nchi za Kiarabu kufanya kazi zisizohitaji masomo ya juu. Walisema kuwa lugha hizi mbili zikiwekwa kwenye mtalaa kama masomo ya lazima kwa wanafunzi wote zingewafaa zaidi kuliko Kiswahili. Wao hawakuona kabisa umuhimu wa lugha ya Kiswahili. Jambo hili lilimpelekea mtafiti kukusanya maoni ya walimu katika shule za sekondari ili kubaini kama Kiswahili kinaweza kuwa na manufaa kwa nchi Uganda.

\section{Mahali pa Utafiti}

Utafiti huu uliendelezwa katika Wilaya ya Bududa nchini Uganda. Utafiti ulihusisha wahojiwa 100. Wahojiwa walikuwa walimu katika shule za sekondari zinazopatikana katika Wilaya ya Bududa. Kuna shule 12 za sekondari katika wilaya hii. Hata hivyo, mtafiti aliendeleza utafiti wake katika shule 10 ambazo aliweza kuzifikia kwa urahisi. Shule hizo 10 zilikuwa zile za: Bududa, Bulucheke, Bushika, Bumayoka, Bukalasi, Bubiita 
seed, Shitumi, Nalwanza, Bubiita Comprehensive na Bukigai. Katika kila shule, mtafiti aliteua walimu wa kike tano na walimu wa kiume tano kimakusudi. Kwa jumla mtafiti alikuwa na wahojiwa 100 wakiwemo wahojiwa 50 wa kike na 50 wa kiume.

\section{MAPITIO YA MAANDISHI}

Kwa mujibu wa Bourdieu (2015), maneno 'lugha na uchumi' yanaweza kumaanisha hali ya kuangazia athari za lugha kwenye nyanja mbalimbali za kiuchumi au kuangazia athari za uchumi kwenye lugha hasa katika kuunda mitindo ya kujieleza au uteuzi wa lugha katika tanzu maalumu za kiuchumi. Hii inamaanisha kwamba lugha na uchumi huathiriana; yaani lugha inaweza kuathiri uchumi kwa upande mmoja na uchumi kuathiri lugha kwa upande mwingine.

Kwa mujibu wa Heller (2003), uchumi wa kimataifa una mambo mawili. Jambo la kwanza ni lile la kuwepo kwa mikakati ya kukuza uchumi pamoja na mtaji katika ngazi ya kimataifa. Jambo la pili ni kuwepo kwa watu na maandalizi katika mikakati hiyo. Mambo haya yanastahili kuwasiliana haraka na kwa njia nzuri ili kuleta maafikiano na kutimiza malengo lengwa. Katika mawasiliano haya, sio teknolojia mpya pekee ndiyo iliyo muhimu lakini lugha pia ambayo hutofautiana kutokana majina ya bidhaa na huduma mbalimbali. Hii ni kwa sababu bidhaa na huduma huwa na majina tofautitofauti. Ni kweli kwamba bila lugha, udhibiti wa bidhaa na huduma usingewezekana. Kulingana na mtaalamu huyu, lugha ni kifaa muhimu sana cha mawasiliano kinachosaidia binadamu katika mazingira ya kung'ang'ania biashara. Heller (2003) asisitiza kwamba lugha huunganisha jamii na kukuza uchumi wa nchi. Wingi lugha huleta ushirikiano baina ya nchi au watu na kuwawezesha kuwa na soko moja kama vile soko moja la nchi za Ulaya. Aidha, wwingi lugha huwezesha wafanyakazi kupata kazi katika nchi za kigeni. Hali hii inawezesha usafirishaji wa bidhaa, mtaji na wafanyakazi baina ya nchi tofautitofauti. Mambo haya huchangia ukuaji wa uchumi wa nchi.

Mkwinda-Nyasulu (2013) ananena kuwa dhima ya lugha katika ukuzaji wa uchumi inabainika wazi. Aeleza kwamba, pale ambapo hakuna lugha, maendeleo hukosekana. Hata hivyo, uwepo kwa lugha pekee hakutimizi lengo la kimsingi la lugha ambalo ni mawasiliano. Mkwinda-Nyasulu (2013) anasisitiza kuwa mawasiliano mazuri huleta maendeleo. Mawasiliano ni jambo la kimsingi katika maendeleo na hutekelezwa kupitia lugha. Kwa hivyo, lugha inastahili kuonekana inawasilisha maana lengwa ili kutimiza malengo wala si kuwa lugha tu. Malengo yanaweza kuwa muungano wa taifa na maendeleo ya kiuchumi, umoja na upataji wa elimu. Watu wanaoungana kwa kuzungumza lugha moja huwa na mtazamo sawa; hivyo basi, ni kweli kwamba lugha moja ni muhimu katika kuunganisha watu wenye mtazamo moja.

Kwa mujibu wa Mkwinda-Nyasulu (2013), kuanzia mwaka wa 1960, kumekuwepo na tafiti ambazo zilidhamiria kutoa maelezo kuhusu umuhimu wa lugha kwenye uchumi. Tafiti hizo zimejikita katika athari za lugha kwenye malipo ya wafanyakazi au kupendekeza mikakati ya kiuchumi wakati wa kuteua mkabala na kutathimini sera za lugha. Mkwinda-Nyasulu (2013) kama anavyowanukuu Arcand (1996) anasema lugha huwa inapunguza gharama katika biashara endapo washiriki wanazungumza lugha moja. Jambo hili hupelekea biashara kuongezeka na hivyo ukuaji wa uchumi. Anaendeleza kwamba lugha humwezesha mwajiri kuafikiana na mfanyakazi wake vizuri. Wao hufikia maafikiano bila tashwishi na kuelewana kwa urahisi. Lugha hupanua soko, huchangia ukuaji wa uchumi, ajira na hali ya kung'ang'ania biashara. Mataifa yanasaini mikataba ya kibiashara kutokana na kuwepo kwa lugha. Bila kuwepo na lugha, maraisi wa nchi zinazotaka kushirikiana katika masuala ya biashara wasingeweza kuelewana, jambo ambalo lingewafanya washindwe kuzindua mikataba kama vile jumuiya ya Afrika Mashariki.

Liu na Pizzi (2018) wananong'oa umuhimu wa kuhamasisha watu kuhusu maendeleo ya jamii. Hata hivyo anasema kuwa jamii inaweza kuhamasishwa endapo kuna mawasiliano kamili. Mawasiliano hukamilika kutokana na matumizi mazuri ya lugha. Paulinus anasema kuwa lugha huwezesha watu kushughulika na mambo ya maendeleo ya jamii zao kwa pamoja. Bodomo (1996) na Trudgel (2009) wanasema kuwa lugha ni muhimu sana katika ukuzaji wa uchumi lakini watu hupuuza dhima ya lugha ndogondogo kubeba 
taarifa, thamani ya mawasiliano, mitazamo ya watu, hisia za watu, na kudhihirisha nguvu za lugha katika kuleta maendeleo. Noah (1999) anasema dhima hii ya lugha huwa inapuuzwa na watu wengi kwa sababu watu huwa wanaelewa vibaya dhana ya maendeleo.

Kulingana na Grenier (2015), utafiti kuhusu masuala ya uchumi unaonyesha kuwa umilisi wa lugha moja maarufu ni muhimu sana na huleta ufanisi wa kiuchumi na huwekea uchumi wa nchi msingi thabiti; hata hivyo, Grenier (2015) anasema kwamba kuwepo kwa lugha nyingi katika nchi pia kuna manufaa yake, lugha hudhihirisha utamaduni wa watu. Kwa mujibu wa Grenier (2015), lugha moja maarufu huwezesha watu kuwasiliana na wenzao katika eneo moja au nchi moja. Anasema kuwa kujifunza lugha moja maarufu ni uwekezaji mzuri. Grenier (2015) anasema kuwa uchumi wa nchi huwa unaimarika endapo wananchi wanazungumza lugha moja. Anasema kwa mfano, uzalishaji wa bidhaa huhitaji watu wafanye kazi kwa pamoja na jambo hili huwezekana kama watu wote wanaohusika wanazungumza lugha moja. Grenier (2015) anaendelea kusema kuwa shughuli ya ununuzi na uuzaji wa bidhaa pia hutekelezwa kama mnunuzi na mwuzaji wanaelewana kilugha. Kulingana na maelezo ya Grenier (2015), kuwa na hali nzuri kiuchumi huwezekana kama watu katika nchi wanazungumza lugha moja. Anasema kuwa kujifunza lugha moja maarufu ni uwekezaji katika mtaji wa binadamu. Anasema watu wanaojifunza lugha moja maarufu huwa na manufaa wanayopata kuliko wenzao wanaozungumza lugha zao za mama.

Kulingana na Hardach (2018), wananchi kuzungumza lugha nyingi ni muhimu kwa uchumi. Anasema kuwa nchi zenye lugha nyingi huwa zinapata mambo mengi mazuri kama vile kuwa na mauzo mazuri katika masoko ya kimataifa na kuwa na wananchi ambao ni wafanya kazi shupavu. Hardach (2018) anaendelea kusema kuwa lugha ni muhimu sana kitaifa na katika biashara ndogondogo. Lugha ndogondogo hupelekea wananchi kufanikiwa katika biashara zao na hivyo kuimarisha uchumi wa nchi. Hii ni kwa sababu watu wa makabila tofautitofauti huweza kuendeleza biashara zao bila matatizo. Wanunuzi wanaweza kutoa maoni yao kuhusu bei ambayo wameambiwa na wauzaji vilele huwa na uwezo wa kutetea bei ya bidhaa zao. Jambo hili humwacha kila mmojawao akiwa ameridhika. Yaani, mnunuzi huwa ameridhika na mwuzaji vile vile; hii ni kwa sababu wameelewana kilugha. Kwa hiyo, lugha ndogondogo ni muhimu sana katika kuendeleza biashara na hivyo; kujenga uchumi wa nchi.

Kiango (2002) anasema kuwa maingiliano ya jamii zenye tamaduni na lugha mbalimbali hutokana na ushirikiano katika shughuli za biashara na nyinginezo za kijamii. Shughuli hizo huzua haja ya kuwepo kwa lugha moja itakayoweza kuwaunganisha watu wenye tamaduni na lugha mbalimbali. Mwingiliano kama huo huweza kuwa katika viwango mbalimbali. Kiwango cha kwanza ni kile cha mtu binafsi ambapo mtu hutoka kwenye jamii yake na kwenda kuishi kwenye jamii inayozungumza lugha tofauti na ile ya kwao. Katika hali kama hiyo, mtu huyo itabidi ajifunze lugha ya jamii ile ya pili ili aweze kuwasiliana nao. Kiwango cha pili ni kile kinachohusisha mkusanyiko wa watu waliotoka kwenye tamaduni mbalimbali na hawana hata lugha moja ya kuwaunganisha. Watu kama hawa wakikaa pamoja huweza kuzusha lugha ya kati ambayo katika hatua za mwanzo itajulikana kama pijini na baadaye kama krioli. Kwa mujibu wa Kiango (2002), kiwango cha tatu ni maingiliano ya watu wenye tamaduni na lugha mbalimbali husababisha kuteuliwa kwa lugha moja miongoni mwa lugha zao na kutumika kama chombo cha mawasiliano na cha kuziunganisha jamii zote husika. Kiwango hiki chaweza kujitokeza katika ngazi ya wilaya, mkoa, nchi, kanda, bara na hata ulimwengu mzima. Wakati wa enzi ya ukoloni katika Afrika Mashariki, haja ya kuwepo kwa lugha moja itakayotumika hususani katika elimu katika nchi zote Afrika Mashariki zilizokuepo choni ya Mwingereza wakati ule, yaani Tanganyika, Kenya, Uganda ilijitokeza. Matokeo ya haja hii ni kuchaguliwa kwa Kiswahili, hususani lahaja ya Kiunguja itumike kama lugha ya kufundishia mashuleni. Vilevile, matokeo ya haja hiyo yalikuwa ni kuundwa kwa Kamati ya Lugha ya Afrika Mashariki mnamo mwaka 1925 na kuanza kazi mwaka 1930 iliyopewa mamlaka ya kuisanifisha lugha ya Kiswahili, ili kuwe na namna moja rasmi ya kusema na kuandika katika Afrika Mashariki. Baada ya nchi za Afrika Mashariki kupata Uhuru, haja ya kuwepo kwa lugha moja kwa kila nchi kwa ajili ya mawasiliano na wananchi na katika 
shughuli mbalimbali za kiserikali na za kijamii ikajitokeza.

\section{Hoja Kuu Ambazo Zimejitokeza Katika Maandiko Mbalimbali}

Jedwali la 1: Hoja Kuu Ambazo Zimejitokeza Katika Maandiko Mbalimbali

\begin{tabular}{|c|c|}
\hline & Hoja \\
\hline 1 & $\begin{array}{l}\text { Lugha hudhibiti bidhaa na huduma kwa } \\
\text { kutoa majina. }\end{array}$ \\
\hline 2 & $\begin{array}{l}\text { Mawasiliano mazuri ya lugha huleta } \\
\text { maendeleo. }\end{array}$ \\
\hline 3 & $\begin{array}{l}\text { Lugha huwezesha wafanya biashara } \\
\text { kung'ang'ana na wenzao sokoni. }\end{array}$ \\
\hline 4 & $\begin{array}{l}\text { Lugha hukuza umoja wa wananchi na } \\
\text { kuleta ukuaji wa uchumi wa nchi. }\end{array}$ \\
\hline 5 & Lugha hupamba matangazo ya biashara. \\
\hline 6 & $\begin{array}{l}\text { Lugha huwezesha wafanya biashara } \\
\text { kutambua masoko ndani na nje ya nchi. }\end{array}$ \\
\hline 7 & $\begin{array}{l}\text { Lugha hupunguza gharama ya kufanya } \\
\text { biashara. }\end{array}$ \\
\hline 8 & $\begin{array}{l}\text { Lugha hupelekea wafanya biashara } \\
\text { kuafikiana. }\end{array}$ \\
\hline 9 & $\begin{array}{l}\text { Lugha hutumiwa katika kuelimisha } \\
\text { wananchi juu ya miradi mbalimbali ya } \\
\text { serikali ya kukuza uchumi wa nchi. }\end{array}$ \\
\hline 10 & $\begin{array}{l}\text { Lugha huwezesha serikali kutia sahihi } \\
\text { mikataba ya kibiashara na mataifa ya } \\
\text { kigeni. }\end{array}$ \\
\hline 11 & $\begin{array}{l}\text { Lugha huwezesha wafanya biashara } \\
\text { kuingia sokoni bila hofu. }\end{array}$ \\
\hline
\end{tabular}

Kulingana na Jedwali la 1 inabainika wazi kuwa lugha huwa inasaidia wafanya biashara kudhibiti, bidhaa na huduma wanazoziuza kwa uma. Hii ni kwa sababu bidhaa na huduma hudhibitiwa kwa kupewa majina tofauti. Bila shaka majina hutokana na kuwepo kwa lugha. Kwa hiyo, Kiswahili kama lugha huchangia jambo hili jinsi kuna majina tofautitofauti ya Kiswahili. Kudhibiti kwa bidhaa na biashara huwa kunapelekea wafanya biashara, makampuni, na serikali kupata faida badala ya kupata hasara. Jambo hili huwa linapelekea uchumi wa nchi kukua.

Kwa mujibu wa Jedwali la 1, mawasiliano mazuri huleta maendeleo ya nchi. Hii ni kwa sababu biashara baina ya watu na nchi hunawiri na kuimarika kutokana na mawasiliano mazuri. Ukosefu wa mawasiliano mazuri baina ya nchi na watu huwa unaathiri biashara. Kwa hiyo, Kiswahili kama lugha kikitumiwa vizuri kinaweza kupelekea biashara kuimarika baina ya nchi na baina ya jamii. Kwa mfano, mawasiliano mazuri kwa kutumia Kiswahili yanaweza kuimarisha biashara baina ya nchi za Afrika Mashariki na baina ya watu wa nchi hizo.

Jedwali 1 laonyesha kwamba lugha huwezesha wafanya biashara mbalimbali kung'ang'ania biashara sokoni. Kama ilivyo kawaida, jambo la kimsingi katika biashara ni kupata faida wala si hasara. Kwa hiyo wafanyabiashara hujaribu iwezekanavyo kuona kwamba wanapata wateja. Lugha ni kitu mojawapo kinachovutia wateja. Lugha nzuri huwavutia wateja na lugha chafu huwafukuza. Kwa hiyo, wafanyabiashara wanaotumia Kiswahili wakiitumia vizuri wanaweza kuendeleza biashara zao na kukuza uchumi wa nchi zao.

Kwa kurejelea Jedwali la 1, tunaona kwamba lugha huwa inaleta umoja miongoni mwa watu na kukuza uchumi wa nchi. Hii ni kwa sababu umoja huleta amani. Nchi yenye amani huwa na maendeleo ya kiuchumi kinyume cha nchi isiyo na umoja huwa na migogoro na vita, mambo ambayo yanapelekea uchumi wa nchi kuporomoka. Kwa hiyo, Kiswahili kama lugha kikitumiwa vizuri kinaweza kuleta umoja miongoni mwa watu wa Afrika Mashariki na kukuza uchumi wa hizi.

Tukitazama Jedwali la 1, inabainika wazi kuwa lugha huwa inatumiwa katika kupamba matangazo ya biashara. Utangazaji ni muhimu sana katika biashara. Lugha inayotumiwa katika matangazo ikipambwa vizuri hupelekea bidhaa husika kuuzwa. Bidhaa zikiuzwa kwa wingi hukuza uchumi wa nchi. Kwa hiyo, lugha ya Kiswahili ikitumiwa vizuri katika matangazo ya kibiashara inaweza kuchangia pakubwa sana ukuaji wa uchumi wa nchi za Afrika Mashariki. 
Jedwali la 1 linaonyesha kuwa lugha huwa inasaidia katika kutambua masoko ndani na nje ya nchi. Wafanyabiashara huwa wanatambua masoko kwa kutumia lugha. Bila kuwepo kwa lugha, watu wasingeweza kuwasiliana na ingekuwa vigumu wao kutambua kuliko masoko. Lakini kutokana na kuwepo kwa lugha, wafanya biashara huwa wanatambua masoko katika wilaya, mikoa na nchi tofauti. Kwa hiyo, lugha ya Kiswahili inaweza kusaidia wafanya biashara katika nchi za Afrika Mashariki kutambua masoko katika jamii mbalimbali.

Hali kadhalika, Jedwali la 1 linaonyesha kuwa lugha huwa inapunguza gharama za kufanya biashara. Hapa tunaona kuwa mfanya biashara asiyeelewa lugha husika huhitaji huduma za mtafsiri. Ili atumie, mfasiri inambidi amlipe. Jambo hili hupandisha gharama ya kufanya biashara. Kwa upande mwingine, mfanya biashara anayeelewa lugha huendeleza biashara yake bila usaidizi wa mfasiri. Jambo hili hupunguza gharama ya kufanya biashara. Kwa hiyo, lugha ya Kiswahili inaweza kusaidia wakazi wa Afrika Mashariki kufanya biashara zao katika nchi zote kwa gharama ya chini ikilinganishwa na wafanya biashara wa kigeni.

Zaidi ya hayo, Jedwali la 1 linaonyesha kuwa lugha huwa inapelekea wafanya biashara kuafiakiana katika mapatano yao. Kama ilivyo kawaida, bidhaa huuzwa baada ya mnunuzi na muuzaji kuafikiana juu ya bei. Wakati mwingine jambo hili huwa gumu sana na lugha ndiyo inayotatua tatizo linavyojitokeza. Muuzaji huwa anauza bidhaa yake kwa bei ya juu kutokana na matumizi yake ya lugha yenye ushawishi. Kutokana na matumizi ya lugha, bidhaa yenye thamani ya chini inaweza kuuzwa kwa bei ya juu zaidi. Faida inayotokana na bidhaa duni kuuzwa kwa bei ya juu zaidi huimarisha uchumi wa nchi. Kwa hiyo, lugha ya Kiswahili inaweza kupelekea wafanya biashara kupata faida kutokana na biashara zao na hivyo kuchangia ukuaji wa uchumi wa nchi.

Jedwali la 1 vilevile linadhihirisha kuwa lugha huwa inatumiwa kuelimisha watu kuhusu miradi mbalimbali ya serikali. Mara nyingi serikali huanzisha miradi ya kuleta maendeleo katika nchi zao. Miradi hiyo hudhamiria kuimarisha uchumi wa nchi. Hii ni kwa sababu uchumi ndio msingi wa maendeleo ya nchi. Kwa hiyo, ili wananchi wakumbatie miradi inayoanzishwa na serikali, wao hustahili kuelimishwa kwanza. Bila kufanya hivyo, miradi husika haiwezi kufaulu. Hii inamaanisha kuwa lugha ya Kiswahili inaweza kutumiwa kuelimisha watu katika nchi za Afrika Mashariki kuhusu miradi inayoanzishwa na serikali kwa madhumuni ya kuleta maendeleo. Hii ni kwa sababu asilimia kubwa ya watu huwa wanazungumza Kiswahili.

Serikali mbalimbali pia huwa zinatia sahihi mikataba ya kibiashara. Lengo la mikataba hiyo ni kuimarisha uchumi wa nchi zao. Mataifa machanga huwa yakitia sahihi mikataba na mataifa waliyoendelea kama vile Marekani, Uingereza, Ufaransa, Ujerumani, Urusi, Ujapani na Uchina. Lugha huwa na mchango mkubwa sana katika mikataba hio. Hii ni kwa sababu lugha huwa inaleta maafikiano baina ya viongozi husika. Vipengele mbalimbali hufafanuliwa kwa kutumia lugha. Ni kweli kwamba lugha ya Kiswahili huwa inatekeleza jambo hili kwa wafanya biashara katika nchi za Afrika Mashariki. Bila shaka kuna watu katika nchi zetu ambao wanatia sahihi mikataba ya kibiashara kwa kutumia lugha ya Kiswahili. Kwa hiyo, lugha hii huwa inachangia pakubwa sana ukuaji wa uchumi wa nchi husika.

Mwisho, lugha hupelekea wafanya biashara kuingia sokoni bila hofu. Hii ni kwa sababu wafanya biashara wenye umilisi wa lugha huwa wanaendeleza biashara zao bila kuwa na wasiwasi. Wao huwa wanajiamini na wanajumuika na wenzao pale Sokoni bila hofu. Hali hii huwafanya wapate faida katika biashara zao. Ni kweli kwamba kuna wafanya biashara wengi sana katika nchi za Afrika Mashariki wanaoendeleza biashara zao kwa kutumia lugha hii. Wachuuzi wengi huwa wanatumia lugha ya Kiswahili kuendeleza biashara zao. Kwa hiyo, lugha ya Kiswahili imechangia pakubwa sana ukuaji wa uchumi wa nchi za Afrika Mashariki. 


\section{MATOKEO YA UTAFITI}

\section{Jedwali la 2: Idadi ya Majibu kwa Kila Hoja}

\begin{tabular}{|c|c|c|}
\hline Hoja Zilizotolewa & Majibu & Asilimia \\
\hline $\begin{array}{l}\text { Watu kupata kazi za } \\
\text { Kiswahili }\end{array}$ & 100 & $100 \%$ \\
\hline Walipa ushuru kuongezeka & 100 & $100 \%$ \\
\hline $\begin{array}{l}\text { Kiwango cha ukosefu wa } \\
\text { ajira kupungua }\end{array}$ & 94 & $94 \%$ \\
\hline $\begin{array}{l}\text { Upeo wa soko la bidhaa } \\
\text { kupanuka }\end{array}$ & 96 & $96 \%$ \\
\hline $\begin{array}{l}\text { Faida za wafanya biashara } \\
\text { kuongezeka }\end{array}$ & 93 & $93 \%$ \\
\hline $\begin{array}{l}\text { Watu kuanzisha kazi za } \\
\text { utaalamu }\end{array}$ & 95 & $95 \%$ \\
\hline $\begin{array}{l}\text { Watu kuandika vitabu vya } \\
\text { Kiswahili na kuviuza }\end{array}$ & 100 & $100 \%$ \\
\hline $\begin{array}{l}\text { Watu kufanya biashara bila } \\
\text { kutatizwa kilugha }\end{array}$ & 98 & $98 \%$ \\
\hline $\begin{array}{ll}\text { Kuunganisha } & \text { makabila } \\
\text { mengi yenye } & \text { tamaduni } \\
\text { tofauti } & \end{array}$ & 100 & $100 \%$ \\
\hline $\begin{array}{l}\text { Kupunguza gharama za } \\
\text { kufanya biashara }\end{array}$ & 85 & $85 \%$ \\
\hline $\begin{array}{l}\text { Kustawisha tamaduni za } \\
\text { Waganda }\end{array}$ & 58 & $58 \%$ \\
\hline $\begin{array}{l}\text { Kupanua kiwango cha } \\
\text { maadili }\end{array}$ & 64 & $64 \%$ \\
\hline $\begin{array}{l}\text { Wafanyakazi wa serikali } \\
\text { kuweza kuwasiliana na } \\
\text { wananchi } \\
\text { hawajasoma }\end{array}$ & 93 & $93 \%$ \\
\hline $\begin{array}{l}\text { Kuibua kizazi kipya } \\
\text { kinachozungumza } \\
\text { kiswahili }\end{array}$ & 74 & $74 \%$ \\
\hline
\end{tabular}

Uchanganuzi na uwasilishaji wa data umetokana na taarifa zilizopo katika Jedwali la 2 hapo juu.
Jedwali hilo linaonyesha hoja mbalimbali ambazo wahojiwa waliulizwa, majibu yaliyotolewa na wahojiwa hao na asilimia za majibu hayo.

Kwa mujibu wa Jedwali la 2, wahojiwa 100 (100\%) walikubali kuwa ufundishaji wa Kiswahili katika shule za sekondari nchini Uganda unaweza kupelekea wananchi wengi kupata kazi hasa katika nyanja ya Kiswahili. Walisema watu kama hao hasa vijana wanaweza kuajiriwa kama walimu, wanahabari na kama wahudumu wa hotelini. Hakukuwa na mhojiwa hata mmoja ambaye alipinga hoja hiyo. Wahojiwa 100 (100\%) vile vile walikubali kuwa ufundishaji wa Kiswahili katika shule za sekondari nchini Uganda ukiimarika utapelekea ushuru wa nchi kuongezeka na hivyo uchumi kukua zaidi. Walisema kwa sababu watu watakaoajiriwa kama walimu wa Kiswahili, wahudumu wa hotelini na wanahabari wataongeza idadi ya walipa ushuru.

Kulingana na Jedwali la 2, wahojiwa 94 wakiwakilisha asilimia $94 \%$ walidai kuwa ufundishaji wa Kiswahili nchini Uganda ukifanywa kuwa somo la lazima kwa wanafunzi katika shule za sekondari utafanya tatizo la ukosefu wa ajira kupungua. Walisema kwa sababu vijana watakapomaliza masomo yao tu hivi, wataajiriwa kama walimu jinsi kuna watu wachache sana ambao wamehitimu katika lugha hii nchini Uganda. Wahojiwa 96 (96\%) walisema kuwa ufundishaji wa Kiswahili nchini Uganda ukifanywa kuwa somo la lazima kwa wanafunzi wote, utapanua upeo wa soko la bidhaa za Waganda. Wahojiwa hao walieleza kuwa kwa sasa, hasa katika eneo la Bududa, kuna watu wachache sana wanaopeleka bidhaa zao Kenya na Tanzania. Walisema idadi hii ndogo inatokana na watu kutoweza kuzungumza lugha ya Kiswahili kwa ufasaha. Walidai pia kuwa Waganda wengi huwa wakiogopa kuzungumza na watanzania wakidai kwamba Kiswahili chao ni kigumu na hakieleweki.

Kwa mujibu wa Jedwali la 2, wahojiwa 93 (93\%) walidai kuwa ufundishaji wa Kiswahili ukiimarika nchini Uganda, upeo wa soko la bidhaa zao utapanuka zaidi na kwamba upeo huo ukipanuka, basi, kipato cha wafanyabiashara kitaongezeka pia. Walisema jambo hili likitokea, basi, hali ya maisha yao itakuwa nzuri kuliko ilivyo sasa. Wahojiwa 95 wakiwakilisha asilimia 95\% walisema kuwa 
ufundishaji wa Kiswahili nchini Uganda utapelekea baadhi ya wananchi kuanzisha kazi za kitaalamu (Jedwali la 2). Walisema baadhi ya wananchi wanaweza kuanzisha kazi za kitaalamu kama vile kusimamia miradi yenye uhusiano na Kiswahili, kuandikia watu tafiti za Kiswahili, barua na mikataba katika Kiswahili.

Tukirejelea Jedwali la 2, tunaona kwamba wahojiwa wote walisema kuwa kufunza Kiswahili nchini Uganda kitawezesha baadhi ya wananchi kujibunia ajira kama vile kuandika vitabu na kuviuza. Walisema wananchi wengine wanaweza kuanzisha makampuni ya kuandika magazeti kwa Kiswahili na kuyauza badala ya kurandaranda mijini wakitarajiwa kuajiriwa. Halikadhalika, wahojiwa 98 (98\%) walisema kuwa ufundishaji wa Kiswahili nchini Uganda utapelekea wafanya biashara wanaoendeleza biashara zao katika mataifa ya Kenya na Tanzania kufanya hivyo bila kutatizwa kilugha. Wahojiwa walisisitiza kuwa kwa sasa, wananchi wanaofanya biashara zao Kenya na Tanzania huwa wakikumbwa na matatizo ya ainaaina kwa kutokuwa na uwezo wa kuzungumza Kiswahili vizuri.

Vile vile, tukiangazia Jedwali la 2, tunaona kwamba wahojiwa 100 (100\%) walisema kwamba ufundishaji wa lugha ya Kiswahili nchini Uganda utasaidia katika kuunganisha makabila mengi yenye tamaduni tofauti. Wahojiwa hao walisema kwamba kuna makabila mengi sana nchini Uganda, kila kabila likiwa na lugha yake na utamaduni wake. Walisema jambo hili limepelekea wananchi kugawanyika kwa misingi ya kikabila. Walisema kuwa kutokana na hali kama hii, Kiswahili kikiimarika nchini Uganda, bila shaka kitaweza kuunganisha makabila hayo. Wahojiwa 85 (85\%) walidai kwamba ufundishaji wa Kiswahili nchini Uganda utanufaisha wafanya biashara kwa kupunguza gharama za kufanyia kazi. Kwa mfano, badala ya kutumia wafasiri wa lugha na kuwalipa, wafanya biashara watakuwa wakiwasiliana na wateja wao moja kwa moja. Walisisitiza kuwa jambo hili likitekelezwa, basi, pesa ambazo zingeenda kwa wafasiri wa lugha, zitabakia zao na hivyo faida watakayo ipata itakuwa kubwa zaidi.

Wahojiwa 58 (58\%) walidai kuwa ufundishaji wa Kiswahili nchini Uganda utastawisha tamaduni mbalimbali za Kiganda. Walieleza kuwa kila kunapotokea mwingiliano wa tamaduni mbalimbali, huwa na mambo mengi na mapya ya kuigwa. Walisema kuwa Waganda wakianza kuzungumza lugha ya Kiswahili kwa ufasaha, bila shaka tamaduni zao zitastawishwa kwa sababu kuna mambo mapya ambayo watajifunza. Aidha, kwa Jedwali la 2, tunaona kwamba wahojiwa 64 (64\%) walisema kuwa ufundishaji wa Kiswahili nchini Uganda utapelekea kiwango cha maadili katika jamii mbalimbali kupanuliwa. Walisema hii ni kwa sababu kuna maadili mapya ambayo wanafunzi watakuwa wakifunzwa shuleni na wanaporudi, wao pia wanaweza kuwafunza wazazi wao.

Wahojiwa 93 (93\%) walieleza kuwa ufundishaji wa Kiswahili nchini Uganda utawawezesha wafanya kazi wa serikali kama vile madaktari na mahakimu kuwasiliana kwa urahisi na wananchi ambao hawakuenda shuleni. Walisema hii ni kwa sababu asilimia kubwa ya Waganda huwa wanazungumza Kiswahili ingawa si sanifu. Walisema kwa hiyo, watu wakifundishwa Kiswahili shuleni, mwishowe wakipata kazi, wataweza kuwasiliana na wananchi bila tatizo. Walisema jambo hili litawawezesha wafanya kazi wa serikali pia kufanya kazi katika maeneo tofauti ya nchi. Mwishowe, wahojiwa 74 (74\%) walidai kuwa ufundishaji wa Kiswahili nchini Uganda utaibua kizazi kipya cha watu wanaozungumza lugha hii. Walisema kwa sababu Kiswahili kikifanywa kuwa somo la lazima kwa wanafunzi wote katika shule za sekondari kisha baadaye vyuo vikuu, shule za msingi na vyuo anuai, basi, bila shaka kutaibuliwa kizazi kipya cha watu wanaozungumza lugha ya Kiswahili kwa ufasaha.

\section{HITIMISHO}

Kwa kuzingatia hoja ambazo zimewasilishwa na kujadiliwa katika makala hii, ni kweli kwamba lugha ya Kiswahili inaweza kuwa na mchango mkubwa sana kwa nchi ya Uganda. Hii inamaanisha kuwa nchi ya Uganda inastahili kuweka mikakati madhubuti ya kuendeleza Kiswahili. Kwa hiyo, tume ya Kiswahili ya Afrika Mashariki ina jukumu la kuona kwamba hatua zinachukuliwa ili kuhakikisha Kiswahili kinatiliwa maanani na nchi mbalimbali za jumuiya. Vile vile, mawazo potovu ambayo yalitolewa na wabunge wa Bunge la Uganda wakati wa kuzindua mtaala mpya 
wa elimu nchini humo yanastahili kupuuzwa na wapenzi wa lugha ya Kiswahili.

\section{MAREJELEO}

Arcand, J. L. (1996). Development economics and language: the earnest search for a mirage? International Journal of the Sociology of Language, 121(1), 119-158.

Bodomo, A. B. (1996). On language and development in Africa: The case of Ghana. Nordic journal of African studies, 5(2), $31-51$.

Bourdieu, P. (2015). Perspectives on Language and Society. In Östman, J. \& Verschueren, J. (Eds), Handbook of Pragmatics (p.1-16). John Benjamins.

Grenier, G. (2015). The value of language skills. IZA World of Labor.

Hardach, S. (2018). Confession with Blue Horses (A Novel) and "On Memory and Narrative in the Work of Charlotte Salomon, Art Spiegelman and Christa Wolf" (Critical Commentary). Doctoral dissertation. Goldsmiths: University of London.

Heller, M. (2003). Globalization, the new economy, and the commodification of language and identity. Journal of sociolinguistics, 7(4), 473492.

Kiango, J. G. (2002). Nafasi ya Kiswahili katika ujenzi wa jamii mpya ya Afrika Mashariki. Nordic Journal of African Studies, 11(2), 13-13.

Liu, A. H., \& Pizzi, E. (2018). The language of economic growth: A new measure of linguistic heterogeneity. British Journal of Political Science, 48(4), 953-980.

Mkwinda-Nyasulu, B. (2013). Role of language in socio-economic development: the semiotics are right. Journal of Humanities, 23(1), 213230.

Noah, P. (1999). From Ethnic Marginalization to Linguistic Cleansing: A Contribution to the National Language Question. In Acts of the 9th
Bi-annual Conference of the Modern Languages Association of Nigeria.

Trudell, B. (2009). Local-language literacy and sustainable development in Africa. International Journal of Educational Development, 29(1), 73-79. 